\title{
CCL3 genotype and current depression increase risk of HIV-associated dementia
}

This article was published in the following Dove Press journal:

Neurobehavioral HIV Medicine

16 November 2009

Number of times this article has been viewed

\section{Andrew J Levine' \\ Elyse J Singer' \\ Janet S Sinsheimer ${ }^{2}$ \\ Charles H Hinkin ${ }^{3}$ \\ Jeanette Papp ${ }^{4}$ \\ Sugandha Dandekar ${ }^{4}$ \\ Allison Giovanelli ${ }^{5}$ \\ Paul Shapshak ${ }^{6}$}

'National Neurological AIDS

Bank, Department of Neurology,

${ }^{2}$ Departments of Biomathematics

and Human Genetics, ${ }^{3}$ Department of

Psychiatry and Biobehavioral Science,

${ }^{4}$ Department of Human Genetics,

University of California Los Angeles,

David Geffen School of Medicine, Los Angeles, CA, USA; ${ }^{5}$ Department of

Psychology, University of California Berkeley, Berkeley, CA, USA;

${ }^{6}$ Department of Medicine (Division of Infectious Diseases and International Medicine), University of South Florida, College of Medicine, Tampa, FL, USA

Correspondence: Andrew Levine National Neurological AIDS Bank, I 1645 Wilshire Blvd., Suite 770, Los Angeles, CA 90025, USA

Tel +l 5624775963

Fax + I 3104737772

Email ajlevine@mednet.ucla.edu
Background: The prevalence of Human immunodeficiency virus (HIV)-associated dementia (HAD) has continued to rise even as incidence has fallen. Several host genetic variants have been identified that modify risk for HAD. However, the findings have not been replicated consistently and most studies did not consider the multitude of factors that might themselves confer risk for HAD. In the current study, we sought to replicate the findings of previous studies in a neurologically and behaviorally well-characterized cohort.

Methods: The sample consisted of $143 \mathrm{HIV}+$ individuals enrolled in the National NeuroAIDS Tissue Consortium (NNTC). Based on consensus diagnosis, 117 were considered neurologically normal upon study entry, and 26 had HAD. Seven single-nucleotide polymorphisms (SNPs) were genotyped within seven genes (CCL2, CCL3, CCL5, interleukin-1 $\alpha$ [IL-1 $\alpha$ ], IL-10, stromal cell-derived factor 1 , and tumor necrosis factor- $\alpha$ ). Logistic regression analysis was used to predict group membership (normal vs HAD), with predictor variables including length of infection, age, current drug dependence, current depression, and genotype.

Results: The two groups were statistically similar with regards to demographic characteristics, current drug use, and disease factors. The HAD group had significantly greater number of individuals with current depression. Only one SNP, rs1130371 within the gene for CCL3, was entered into the analysis as the others showed symmetric distribution between groups. Logistic regression indicated that current depression and CCL3 genotype were significant predictors of HAD. Depression conferred a fivefold greater risk of HAD, while the TT genotype for CCL3 SNP (rs1130371) was associated with twofold risk for HAD.

Conclusion: Depression and CCL3 genotype predicted HAD. The fact that SNPs previously found to be associated with HAD were not in our analysis, and that rs1130371 is in high linkage disequilibrium with neighboring genes indicates that more dense genotyping in significantly larger cohorts is required to further characterize the relationship between genotype and risk for HAD.

Keywords: HIV-associated dementia, NeuroAIDS, CCL3, cytokine, depression, HIV

While the widespread use of highly active antiretroviral therapy (HAART) has resulted in substantial improvements in life expectancy and lowered incidence of human immunodeficiency virus (HIV)-associated dementia (HAD), significant neuropsychological deficits continue to occur at an alarming rate. ${ }^{1-4}$ Studies have shown that even relatively healthy human immunodeficiency virus-positive (HIV+) individuals are at risk for HAD and related disorders, and HAART does not appear to be adequately protective. ${ }^{2}$ Consequently, discovering endogenous and exogenous factors involved in the neuropathogenesis of HAD remains an important area of NeuroAIDS research.

submit your manuscript | www.dovepress.con 
The dysregulation of the host immunologic response to $\mathrm{HIV}$, and subsequent persistent inflammation, are thought to be a key cause of the neuropathological changes underlying HAD. ${ }^{5}$ As part of this process, host immunologic factors including cytokines and chemokines, and their respective receptors, have been implicated. ${ }^{6}$ This has been best exemplified via genetic studies demonstrating that minor genomic alterations can modify the function of these immunologic factors and subsequently alter susceptibility for HIV infection and/or disease progression. For example, early studies demonstrated that a 32-base pair deletion within the gene for Chemokine (C-C motif) receptor 5 (CCR5) resulting in the CCR5delta-32 allele, conferred high resistance to HIV-1 infection when inherited from both parents ${ }^{7}$ while others found that possessing just one allele was associated with slower disease progression and risk for HAD. ${ }^{8-11}$ In the past ten years, a variety of polymorphisms in the genes of other immunologic factors have also been associated with HIV disease progression and risk for HAD. These include RANTES ${ }^{12}$ CCR2, ${ }^{13,14}$ stromal cell-derived factor $1,{ }^{15,16}$ monocyte chemo-attractant protein 1 (MCP-1), ${ }^{17}$ interleukin-2 (IL-2), ${ }^{18}$ IL- $1 \alpha,{ }^{19}$ and tumor necrosis factor- $\alpha$ (TNF-a), ${ }^{20,21}$ some of which are considered acquired immunodeficiency syndrome (AIDS) restriction genes (ARGs) due to their effect on HIV susceptibility and modulation of disease progression. Although these findings have not been consistently replicated, they have led to a deeper understanding of the role host immunologic factors in HAD, and demonstrated the value of candidate gene studies in elucidating this process.

Because of the complex interactions between genotype and environment, it is also necessary to consider the various exogenous factors that can contribute to vulnerability for HAD. Thus far, the most commonly reported risk factors for HAD include older age, certain medical comorbidities, and substance abuse. Age has been found to be an important risk factor for $\mathrm{HAD},{ }^{3,22,23}$ a factor of increasing importance given that HIV+ individuals are increasingly living beyond their 60s. Comorbid medical conditions that confer risk for HAD include hepatitis $\mathrm{C}$ coinfection, ${ }^{24,25}$ diabetes, ${ }^{26}$ and anemia. ${ }^{27}$ Finally, illicit drug use also appears to confer risk for HAD. For example, stimulant use may act synergistically or additively with HIV in causing neurocognitive deficits. ${ }^{28}$ Notably, while these factors have been widely recognized as contributory for HAD, they have often been overlooked in previous genetic association studies.

In the current paper, we describe a candidate gene association study that examined the contribution of polymorphisms within immunologically related genes to risk for
HAD. This was conducted with a large, well-characterized cohort representative of those currently most affected by this epidemic in the United States. In addition, we considered the contribution of age, ethnicity, current major depression, and current drug use in our statistical models. Our goal was to attempt to replicate and expand upon the findings of previous candidate gene studies by including important exogenous and endogenous co-factors and by utilizing a cohort that was well-characterized neurocognitively and neurologically.

\section{Methods}

\section{Participants}

Data and tissue/fluid samples were collected from HIV+ individuals enrolled in the National NeuroAIDS Tissue Consortium (NNTC) according to Institutional Review Board (IRB)-approved procedures. The NNTC is a multicenter, longitudinal study of well-characterized adults with HIV/ AIDS. Subjects are followed longitudinally until death, and a rapid autopsy is performed to extract tissues for research. ${ }^{29}$ The NNTC consists of four sites within the United States: 1) The National Neurological AIDS Bank located in Los Angeles, CA, 2) the Texas Repository for AIDS Research located in Galveston, TX, 3) the Manhattan HIV Brain Bank located in New York, NY, and 4) the California NeuroAIDS Tissue Network located in San Diego, CA. Upon study entry at one of the four sites, participants are administered a comprehensive battery of psychometric measures in order to determine past and current substance use disorders, psychiatric illness, and neuropsychological functioning. Neurological examinations were also performed along with immunological (CD4+ subsets) and virologic (plasma and cerebrospinal fluid HIV viral load) testing.

Only those individuals who met the following criteria were included in the current study: no history of central nervous system (CNS) opportunistic infections (including cryptococcal meningitis, toxoplasmosis, and progressive multifocal leukoencephalopathy), no history of traumatic brain injury, no history of learning disability or other developmental disorders, no other major neurologic syndromes (eg, epilepsy, multiple sclerosis, Parkinson's disease, brain tumor). Further, based on the consensus diagnosis ${ }^{30}$ based on neurologic, neuropsychological, and medical examination, only those individuals who were diagnosed as neurologically normal or who had possible or probable HAD at the time of study entry were included. This was done in order to exclude individuals who may have other etiologies for neurocognitive impairment. Of approximately 2200 NNTC participants, this left 467 eligible for inclusion. Additional 
exclusions were made based on the quality of genotyping (described below), leaving a sample size of 343. Of these, 143 subjects had complete data including psychiatric interview, neuropsychological testing, neurologic diagnosis, and virologic data. The average age of this final sample was 44 $(\mathrm{SD}=7.8)$, with a range of 22 to 65 years. Fifteen $(10.5 \%)$ were female. Thirty-six (25.2\%) were African American, the remaining 92 (64.3\%) were European American.

\section{Neurologic diagnoses}

Using established criteria, ${ }^{30}$ diagnosis of HAD was determined via consensus agreement between the examining study neurologist and neuropsychologist, with consideration of laboratory results (eg, viral load and CD+ T-cell count), neuroimaging (when available), neuropsychological and psychiatric test results, and demographic information. In order to standardize the neurological diagnosis, all NNTC sites used diagnostic work sheets which provide an algorithmic approach to the diagnosis. Those individuals who were diagnosed as neurologically normal or who demonstrated very mild neuropsychological deficits not consistent with HIV-associated neurocognitive disorders were classified as non-impaired, while those who met criteria for possible or probable HAD were classified as HAD.

\section{Psychiatric diagnoses}

The Psychiatric Research Interview for Substance and Mental Disorders (PRISM), ${ }^{31}$ was administered to participants in order to determine history of substance use disorder and major depressive disorder (MDD). The PRISM is a structured diagnostic interview that yields Diagnostic and Statistical Manual of Mental Disorders Fourth Edition. (DSM-IV) diagnoses. Participants were classified as 'currently depressed' if they met diagnostic criteria for current MDD. Participants were classified with none, past, or current substance abuse and/or dependence across a number of common drugs of abuse. For the current study individual were classified as 'drug using' if they reported current dependence on cocaine, heroin, or methamphetamine. Individuals with no current dependence on these substances, or who reported only past dependence on these substances ( $>12$ months prior to study entry), were classified as 'nondrug-using'.

\section{Duration of infection}

Also considered in our analysis was the estimated length of HIV infection. This was based on the participant's selfreported year of infection. In most instances the actual date of infection could not be determined.

\section{Tissue processing, DNA extraction, and genotyping}

Peripheral blood mononuclear cells (PBMCs) and/or frozen tissue samples were shipped to the University of California Los Angeles (UCLA) Biological Samples Processing Core (BSPC) from the four NNTC sites for DNA extraction. The Autopure LS $^{\mathrm{TM}}$ nucleic acid purification instrument was used for extracting DNA. Samples were quantitated using OD 260/280. Extracted DNA was then sent to the UCLA Genotyping Core for genotyping. Prior to genotyping, the samples were checked for concentration by Quant-iT ds DNA Assay kit (Invitrogen, Carlsbad, CA) and for quality by agarose gel. DNA amplification was performed on 96 and 384-well polymerase chain reaction (PCR) plates on GeneAmp PCR System 9700 thermal cyclers (Applied Biosystems, Foster City, CA). Single nucleotide polymorphism (SNP) genotypes were determined using the allelic discrimination assay on an Applied Biosystems 7900 Taqman instrument analyzed with SDS2.3 software. These SNPs are listed in Table 1. Three SNPs (rs1719130, rs1024611, rs1130371) occurred on the same chromosome but were not found to be in significant linkage disequilibrium. Data then underwent error-checking and data-cleaning including control checks, duplicates checks, and checking for Hardy-Weinberg equilibrium. Each genotype was evaluated independently according to a number of quality parameters. Data from cases with genotyping success rate of $<75 \%$ were removed. (Note that brain tissue had poorer genotyping success rate compared to PBMC [ $74 \%$ vs $88 \%$, respectively], and those with HAD upon study entry were more likely to have been genotyped with brain tissue. Therefore, the HAD group had higher genotyping failure rate.) Polymorphisms were not

Table I Allelic distribution by group

\begin{tabular}{|c|c|c|c|c|}
\hline \multirow[t]{3}{*}{ SNP (Gene) } & \multicolumn{2}{|c|}{ Homozygous frequency } & \multirow[t]{3}{*}{$\chi^{2}$} & \multirow[t]{3}{*}{$P$-value } \\
\hline & Minor allele & Major allele & & \\
\hline & $\begin{array}{l}\text { Non-impaired/ } \\
\text { HAD }\end{array}$ & $\begin{array}{l}\text { Non-impaired/ } \\
\text { HAD }\end{array}$ & & \\
\hline rsI0246II (CCL2) & $8 \% / 8 \%$ & $47 \% / 44 \%$ & 0.05 & 0.97 \\
\hline rsII3037I (CCL3) & $16 \% / 31 \%$ & $65 \% / 42 \%$ & 4.86 & 0.09 \\
\hline rsI7I9130 (CCL5) & $6 \% / 15 \%$ & $60 \% / 46 \%$ & 3.18 & 0.20 \\
\hline rs|756| (IL-| $\alpha)$ & $4 \% / 4 \%$ & $60 \% / 54 \%$ & 3.54 & 0.84 \\
\hline rs I 800872 (IL-I0) & $12 \% / 4 \%$ & $45 \% / 40 \%$ & 2.12 & 0.35 \\
\hline rsl800629 (TNF- $\alpha)$ & $2 \% / 0 \%$ & $77 \% / 77 \%$ & 0.47 & 0.79 \\
\hline rsI80II57 (SDF-I) & $4 \% / 0 \%$ & $75 \% / 77 \%$ & 1.23 & 0.54 \\
\hline
\end{tabular}

Abbreviations: HAD, HIV-associated dementia; HIV, human immunodeficiency virus; IL, interleukin; SDF-I, stromal cell-derived factor I; SNP, single-nucleotide polymorphisms; TNF- $\alpha$, tumor necrosis factor- $\alpha$. 
included if their genotyping rate was $<75 \%$, or if they did not meet Hardy-Weinberg equilibrium.

\section{Statistical analysis}

Statistical analysis was conducted in two stages. In the first stage, all polymorphisms were entered into individual contingency table analyses to determine their allelic distribution between the non-impaired and HAD individuals unadjusted for other covariates (Table 1). Significance was assessed by calculating the chi-square value with a more liberal $(0.10) P$-value considering the expected direction of the findings. Those that reached statistical significance in this analysis were then entered into the second stage of the statistical analysis that adjusted for potential covariates. This second stage consisted of a logistical regression analysis. For the logistic regression, age (in years), ethnicity (African American vs European American), current drug dependence, current MDD, and length of HIV infection were entered considered as potential covariates in the first step. In the second step, covariates found conditionally to be significant in the first step were entered.

\section{Results}

Groups were compared across a number of measures prior to the logistic regression (see Table 2). Based on chi-square analysis, the groups did not differ with regards to proportion of females $\left(\chi^{2}=0.04, P=0.73\right)$, lifetime IV drug use $\left(\chi^{2}=2.53\right.$, $P=0.17)$, current drug dependence $\left(\chi^{2}=0.48, P=0.69\right)$, or ethnicity $\left(\chi^{2}=1.62, P=0.32\right)$. The HAD group had a significantly greater number of individuals with current MDD $\left(\chi^{2}=6.47, P=0.02\right)$. Length of HIV infection and CD4+ T-cell count were statistically similar $(\mathrm{F}=0.41, P=0.52$ and $\mathrm{F}=0.09, P=0.77$, respectively). Both groups were similar in age $(\mathrm{F}=0.38, P=0.54)$. As expected, the HAD group had significantly greater neuropsychological impairment $(\mathrm{F}=102$, $P<0.001)$.

Of the seven SNPs examined in contingency table analyses, only the frequency of rs1130371 of the MIP1-alpha gene was considered significantly different between the two groups $(P<0.10)$. Specifically, $31 \%$ of the HAD was homozygous for the $\mathrm{T}$ allele, as compared to $16 \%$ of the non-impaired group. This SNP was entered into the second step of the logistic regression. Of the variables entered into the first step, only current $\mathrm{MDD}$ reached significance (odds ratio $[\mathrm{OR}]=5.06, P=0.005$ ). In the second step, rs1130371 was also significant $(\mathrm{OR}=2.04$, $P=0.017)$. The overall model was statistically significant $\left(\chi^{2}=14.47, P=0.025\right)$. Due to the differential distribution of rs1130371 genotype among African Americans and European Americans, an interaction term was entered in a third step. No evidence of a SNP-by-ethnicity interaction was found (Table 3).

\section{Discussion}

This candidate gene study sought to determine if genetic polymorphisms within a variety of genes coding for cytokines and other immunologic factors confer risk for HAD. While previous studies have examined the relationship between genotype and risk for HAD, this study also considered the contribution of exogenous factors (current drug dependence and major depression), endogenous factors (age and ethnicity), and virologic factors (length of infection) to this risk. Further, the cohort used for this study was thoroughly characterized with regards to neurologic diagnoses and neuropsychological functioning.

Current MDD conferred an almost fivefold risk for HAD. Such a finding raises important questions about the association between the neuropsychiatric and neurocognitive

Table 2 Participant characteristics by group

\begin{tabular}{|c|c|c|c|}
\hline \multirow[t]{2}{*}{ Characteristic } & \multirow{2}{*}{$\begin{array}{l}\text { Non-impaired }(n=1 \mid 7) \\
\text { Mean }(\mathrm{SD})\end{array}$} & \multirow{2}{*}{$\begin{array}{l}\text { HAD }(n=26) \\
\text { Mean }(S D)\end{array}$} & \multirow[t]{2}{*}{$P$-value } \\
\hline & & & \\
\hline Age in years & $44(8)$ & $45.1(7.1)$ & 0.54 \\
\hline Global cognition (T-score) & $46(6.2)$ & $31.5(6.3)$ & $<0.001$ \\
\hline Years of HIV infection & II.I (5.4) & $10.3(6)$ & 0.52 \\
\hline CD4+T-cell/mm $/ \mathrm{mm}^{3}$ & 214 (229) & $229(259)$ & 0.77 \\
\hline \multicolumn{4}{|c|}{ Proportion of cases by group } \\
\hline Female & $10.5 \%$ & $11.5 \%$ & 0.74 \\
\hline African American & $25.2 \%$ & $15.4 \%$ & 0.32 \\
\hline History of IV drug use & $17.1 \%$ & $31.8 \%$ & 0.17 \\
\hline Current MDD & $13.7 \%$ & $34.6 \%$ & 0.02 \\
\hline Current drug* dependence & $7.7 \%$ & $3.8 \%$ & 0.69 \\
\hline
\end{tabular}

Notes: *Cocaine, heroin, and/or methamphetamine.

Abbreviations: HAD, HIV-associated dementia; HIV, human immunodeficiency virus; IV, intravenous; MDD, major depressive disorder. 
Table 3 Results of logistic regression analysis

\begin{tabular}{llllll}
\hline Variable & Beta & Standard error & Degrees of freedom & P-value & Exp(B) \\
\hline Length of infection & 0.007 & 0.046 & $\mathrm{I}$ & 0.88 & 0.53 \\
Age & 0.019 & 0.030 & $\mathrm{I}$ & 0.005 & 1.019 \\
Current depression & 1.621 & 0.572 & $\mathrm{I}$ & 5.056 \\
Current drug dependence & -1.227 & 1.187 & $\mathrm{I}$ & 0.301 & 0.293 \\
Race & -0.180 & 0.718 & $\mathrm{I}$ & 0.802 & 0.835 \\
rsII3037I & 0.713 & 0.299 & $\mathrm{I}$ & 0.017 & 2.04 \\
Race-by-genotype & -0.667 & 1.108 & $\mathrm{I}$ & 0.547 & 0.513 \\
\hline
\end{tabular}

manifestations of HIV. Notably, a recent study found incidence of MDD during a two-year period to be associated with symptomatic HIV disease at baseline, as well as prior psychiatric history. ${ }^{32}$ Depression has also been found to be associated with accelerated disease progression. ${ }^{33}$ Such findings suggest an association between depression and the biological processes underlying HAD. Indeed, there may be a common biological pathway leading to HAD and depression. There is now ample evidence to support the hypothesis that pro-inflammatory cytokines released during viral infection are involved in depression. ${ }^{34,35}$ It is plausible that significantly elevated cytokine levels detected in those with $\mathrm{HAD}^{36}$ also result in depression, as detected by the PRISM in the current study. Alternatively, it may be that a more indirect relationship exists between depression and HAD. For example, depression may lead to delayed initiation of HAART and also greater noncompliance. ${ }^{37,38}$ As such, HIV may be allowed more time to penetrate the CNS and proliferate, thus leading to neuropathological changes and ultimately HAD. Still another putative explanation is that those with HAD experience greater rates of reactive depression in response to deteriorating cognitive functioning and loss of independence. This question will need to be further addressed, but our results undoubtedly provide strong evidence for a link between depression and HAD.

The only other variable that offered statistically significant prediction value was the rs1130371 SNP within the CCL3 gene. In our sample, individuals with HAD had almost double the frequency of homozygosity for the nonancestral $\mathrm{T}$ allele ( $31 \%$ versus $16 \%$ ). CCL3 (also commonly called MIP- $1 \alpha$ ) is a natural ligand of the CCR5 HIV co-receptor and may be partially responsible for CD8+ T-cell suppression of HIV..$^{39,40}$ The rs1130371 SNP is a synonymous $\mathrm{C} \rightarrow \mathrm{T}$ substitution at position +868 in exon 2 of the CCL3 gene, ${ }^{41}$ suggesting that it does not alter the function or expression of CCL3. However, it is in high linkage disequilibrium with a variety of nearby SNPs not only in the CCL3 gene, but also the CCL4 and CCL18 genes. ${ }^{41}$ There are few studies describing the role of CCL3 in HIV. Modi and colleagues ${ }^{41}$ genotyped 21 SNPs, including rs1130371, in a 47-kb interval on chromosome 17 containing the genes for CCL3, CCL4 (MIP-1 $\beta$ ), and CCL18 (DC-CK1/ PARC/AMAC-1). Seven highly correlated SNPs spanning the three genes examined were significantly associated with more rapid disease progression among Europeans Americans, but not African Americans. ${ }^{41}$ While rs1 130371 was not among the seven SNPs, it was in high linkage disequilibrium (LD) with some, including those within the CCL4 and CCL18 genes. In another study, Gonzalez and colleagues examined HIV-1 transmission and disease-modifying effects of CCL3 haplotypes based on the rs1719134 and rs35511254 polymorphisms. ${ }^{42}$ Three haplotypes based on these two SNPs were identified, and they varied markedly between the African Americans, European Americans, and Hispanics. For example, the authors reported one haplotype (TT) that was common in African Americans but observed in less than $1 \%$ of European Americans. Among the European Americans in their sample, the length of time before developing AIDS was shorter in individuals lacking the common ancestral haplotype (CC). While no disease-modifying effect was observed for any of the CCL3 haplotypes in African Americans, possession of the TT haplotype among this group was associated with a significantly lower risk of HIV-1 acquisition. In short, the Gonzalez study indicates considerable variation in genotype between races, and differential disease modifying effects depending on ethnicity. Importantly, our analyses did indicate a significantly different distribution of rs1130371 genotypes between African Americans and European Americans. However, after entering an interaction term for ethnicity-by-genotype, our logistic regression was still significant, as was the rs1130371 SNP. Despite this, it is clear from the existing literature on CCL3 that further examination of this stretch of the genome coding for this chemokine is required to determine the specific polymorphism or haplotype that is driving the findings reported here.

The role of CCL3 has also been investigated in other neurodegenerative conditions. A relationship between CCL3 
and Alzheimer's disease (AD) was previously reported. ${ }^{43}$ In fact, the peripheral T-cells of individuals with $\mathrm{AD}$ demonstrate over expression of CCL3, which promotes T-cell transendothelial migration across the blood-brain barrier via binding to CCR5 on brain endothelial cells. ${ }^{44}$ Such phenomena may be particularly germane to unraveling the neuropathogenesis of HAD. Further, like CCL5 (RANTES) and CCL4, CCL3 is a beta-chemokine and natural ligand of CCR5. CCL5 and CCL4 are considered ARGs due to their modulatory effect on HIV/AIDS disease progress. ${ }^{45}$ Like those ARGs, CCL3 may act to slow disease progression by inhibiting HIV entry into the cells within the CNS, or cells that would ultimately enter the CNS.

Several unavoidable factors limit the interpretation and generalizability of the results from this study. First, while our sample size was sufficient for the statistical analysis conducted, it is preferable to have very large samples for genetic studies due to the small effects genotype at a single SNP may have upon measurable phenotypes. However, while small, our sample was very well characterized with regards to the phenotype of interest. Thus, the exclusion of many hundred cases not meeting our strict inclusion criteria would have reduced the overall power given perfect data, but probably strengthened the validity of our findings. Second, we decided to conduct the statistical analyses on a sample of mixed ethnicities to increase our sample size of clean data. This strategy seems justified by the homogeneity of the results we found among the ethnic subsets. Further, while we observed significantly different CCL3 allele frequencies between European Americans and African Americans, our statistical analysis indicated that ethnicity was not a factor in the statistical analysis findings. Fourth, the diagnosis of HAD is often difficult considering the high frequency of comorbid medical and psychiatric conditions in this population. This difficulty was clearly shown in an inter-rater reliability study of HAD diagnosis in this sample, ${ }^{30}$ and supports our choice of strict inclusion criteria. It is hoped that with more recently refined diagnostic nosology for HAD, ${ }^{46}$ this problem will be overcome in future analyses. Finally, as discussed above, the high LD in the area of the genome surrounding rs1130371 will require a follow-up study that genotypes this region more densely.

\section{Acknowledgments}

This study was funded through the California HIV/AIDS Research Program grant ID06-LA-187, of which Dr Levine was the principal investigator. This study was also made possible through grants U01-MH08021 and R24-NS38841 (National Neurological AIDS Bank), U01-MH083507 and
R24-NS45491 (Texas Repository for AIDS Research), U01-MH083501 and R24-MH59724 (Manhattan HIV Brain Bank), U01-MH083506 and R24-MH59745 (California NeuroAIDS Tissue Network).

\section{References}

1. Ances BM, Ellis RJ. Dementia and neurocognitive disorders due to HIV-1 infection. Semin Neurol. 2007;27(1):86-92.

2. Dore GJ, Correll PK, Li Y, Kaldor JM, Cooper DA, Brew BJ. Changes to AIDS dementia complex in the era of highly active antiretroviral therapy. AIDS. 1999;13(10):1249-1253.

3. Valcour V, Shikuma C, Watters M, Sacktor N. Cognitive impairment in older HIV-1-seropositive individuals: prevalence and potential mechanisms. Aids. 2004;18(Suppl 1):S79-S86.

4. Wojna V, Skolasky RL, Hechavarria R, et al. Prevalence of human immunodeficiency virus-associated cognitive impairment in a group of Hispanic women at risk for neurological impairment. J Neurovirol. 2006;12(5):356-364.

5. Cartier L, Hartley O, Dubois-Dauphin M, Krause KH. Chemokine receptors in the central nervous system: role in brain inflammation and neurodegenerative diseases. Brain Res Brain Res Rev. 2005;48(1): $16-42$.

6. Levine AJ, Singer EJ, Shapshak P. The role of host genetics in the susceptibility for HIV-associated neurocognitive disorders. AIDS Behav. 2009;13(1):118-132.

7. Samson M, Libert F, Doranz BJ, et al. Resistance to HIV-1 infection in caucasian individuals bearing mutant alleles of the CCR- 5 chemokine receptor gene. Nature. 1996;382(6593):722-725.

8. Boven LA, van der Bruggen T, van Asbeck BS, Marx JJ, Nottet HS. Potential role of CCR5 polymorphism in the development of AIDS dementia complex. FEMS Immunol Med Microbiol. 1999;26(3-4): 243-247.

9. Meyer L, Magierowska M, Hubert JB, et al. Early protective effect of CCR-5 delta 32 heterozygosity on HIV-1 disease progression: relationship with viral load. The SEROCO Study Group. Aids. 1997;11(11): F73-F78.

10. Singh KK, Barroga CF, Hughes MD, et al; Genetic influence of CCR5, CCR2, and SDF1 variants on human immunodeficiency virus 1 (HIV-1)-related disease progression and neurological impairment, in children with symptomatic HIV-1 infection. J Infect Dis. 2003;188(10): 1461-1472.

11. van Rij RP, Portegies P, Hallaby T, et al. Reduced prevalence of the CCR5 delta32 heterozygous genotype in human immunodeficiency virus-infected individuals with AIDS dementia complex. J Infect Dis. 1999;180(3):854-857.

12. Liu H, Chao D, Nakayama EE, et al. Polymorphism in RANTES chemokine promoter affects HIV-1 disease progression. Proc Natl Acad Sci U S A. 1999;96(8):4581-4585.

13. Smith MW, Dean M, Carrington M, et al. Contrasting genetic influence of CCR2 and CCR5 variants on HIV-1 infection and disease progression. Hemophilia Growth and Development Study (HGDS), Multicenter AIDS Cohort Study (MACS), Multicenter Hemophilia Cohort Study (MHCS), San Francisco City Cohort (SFCC), ALIVE Study. Science. 1997;277(5328):959-965.

14. Singh KK, Ellis RJ, Marquie-Beck J, et al. CCR2 polymorphisms affect neuropsychological impairment in HIV-1-infected adults. J Neuroimmunol. 2004;157(1-2):185-192.

15. Michael NL, Moore JP. HIV-1 entry inhibitors: evading the issue. Nat Med. 1999;5(7):740-742.

16. Winkler C, Modi W, Smith MW, et al. Genetic restriction of AIDS pathogenesis by an SDF-1 chemokine gene variant. ALIVE Study, Hemophilia Growth and Development Study (HGDS), Multicenter AIDS Cohort Study (MACS), Multicenter Hemophilia Cohort Study (MHCS), San Francisco City Cohort (SFCC). Science. 1998;279(5349): 389-393. 
17. Gonzalez E, Rovin BH, Sen L, et al. HIV-1 infection and AIDS dementia are influenced by a mutant MCP-1 allele linked to increased monocyte infiltration of tissues and MCP-1 levels. Proc Natl Acad Sci U S A. 2002;99(21):13795-13800.

18. Shrestha S, Strathdee SA, Galai N, et al. Behavioral risk exposure and host genetics of susceptibility to HIV-1 infection. J Infect Dis. 2006;193(1):16-26.

19. Price P, James I, Fernandez S, French MA. Alleles of the gene encoding IL-1alpha may predict control of plasma viraemia in HIV-1 patients on highly active antiretroviral therapy. Aids. 2004;18(11):1495-1501.

20. Quasney MW, Zhang Q, Sargent S, Mynatt M, Glass J, McArthur J. Increased frequency of the tumor necrosis factor-alpha-308. A allele in adults with human immunodeficiency virus dementia. Ann Neurol. 2001;50(2):157-162.

21. Pemberton LA, Stone E, Price P, van Bockxmeer F, Brew BJ. The relationship between ApoE, TNFA, IL1a, IL1b and IL12b genes and HIV-1-associated dementia. HIV Med. 2008;9(8):677-680.

22. Becker JT, Lopez OL, Dew MA, Aizenstein HJ. Prevalence of cognitive disorders differs as a function of age in HIV virus infection. Aids. 2004;18(Suppl 1):S11-S18.

23. Cherner M, Ellis RJ, Lazzaretto D, et al. Effects of HIV-1 infection and aging on neurobehavioral functioning: preliminary findings. Aids. 2004;18(Suppl 1):S27-S34.

24. Cherner M, Letendre S, Heaton RK, et al. Hepatitis C augments cognitive deficits associated with HIV infection and methamphetamine. Neurology. 2005;64(8):1343-1347.

25. Tozzi V, Balestra P, Lorenzini P, et al. Prevalence and risk factors for human immunodeficiency virus-associated neurocognitive impairment 1996 to 2002: results from an urban observational cohort. JNeurovirol. 2005;11(3):265-273.

26. Valcour V, Shikuma C, Shiramizu B, et al. Diabetes, insulin resistance, and dementia among HIV-1-infected patients. J Acquir Immune Defic Syndr. 2005;38(1):31-36.

27. Qureshi AI, Hanson DL, Jones JL, Janssen RS. Estimation of the temporal probability of human immunodeficiency virus (HIV) dementia after risk stratification for HIV-infected persons. Neurology. 1998;50(2):392-397.

28. Rippeth JD, Heaton RK, Carey CL, et al. Methamphetamine dependence increases risk of neuropsychological impairment in HIV infected persons. J Int Neuropsychol Soc. 2004;10(1):1-14.

29. Morgello S, Gelman BB, Kozlowski PB, et al. The National NeuroAIDS Tissue Consortium: a new paradigm in brain banking with an emphasis on infectious disease. Neuropathol Appl Neurobiol. 2001;27(4):326-335.

30. Woods SP, Rippeth JD, Frol AB, et al. Interrater reliability of clinical ratings and neurocognitive diagnoses in HIV.J Clin Exp Neuropsychol. 2004;26(6):759-778

31. Hasin DS, Trautman KD, Miele GM, Samet S, Smith M, Endicott J. Psychiatric Research Interview for Substance and Mental Disorders (PRISM): reliability for substance abusers. Am J Psychiatry. 1996;153(9): 1195-1201.
32. Atkinson JH, Heaton RK, Patterson TL, et al. Two-year prospective study of major depressive disorder in HIV-infected men. J Affect Disord. 2008;108(3):225-234.

33. Leserman J. Role of depression, stress, and trauma in HIV disease progression. Psychosom Med. 2008;70(5):539-545.

34. Dantzer R, Kelley KW. Twenty years of research on cytokine-induced sickness behavior. Brain Behav Immun. 2007;21(2):153-160.

35. Raison CL, Capuron L, Miller AH. Cytokines sing the blues: inflammation and the pathogenesis of depression. Trends Immunol. 2006;27(1):24-31.

36. Letendre SL, Lanier ER, McCutchan JA. Cerebrospinal fluid beta chemokine concentrations in neurocognitively impaired individuals infected with human immunodeficiency virus type 1. J Infect Dis. 1999;180(2):310-319.

37. Li X, Margolick JB, Conover CS, et al. Interruption and discontinuation of highly active antiretroviral therapy in the multicenter AIDS cohort study. J Acquir Immune Defic Syndr. 2005;38(3):320-328.

38. Gordillo V, del Amo J, Soriano V, Gonzalez-Lahoz J. Sociodemographic and psychological variables influencing adherence to antiretroviral therapy. AIDS. 1999;13(13):1763-1769.

39. Cocchi F, DeVico AL, Garzino-Demo A, Arya SK, Gallo RC, Lusso P. Identification of RANTES, MIP-1 alpha, and MIP-1 beta as the major HIV-suppressive factors produced by CD8+ T cells. Science. 1995;270(5243):1811-1815.

40. Moriuchi H, Moriuchi M, Combadiere C, Murphy PM, Fauci AS. CD8+ T-cell-derived soluble factor(s), but not beta-chemokines RANTES, MIP-1 alpha, and MIP-1 beta, suppress HIV-1 replication in monocyte/macrophages. Proc Natl Acad Sci U S A. 1996;93(26): $15341-15345$.

41. Modi WS, Lautenberger J, An P, et al. Genetic variation in the CCL18-CCL3-CCL4 chemokine gene cluster influences HIV Type 1 transmission and AIDS disease progression. Am J Hum Genet. 2006;79(1):120-128.

42. Gonzalez E, Dhanda R, Bamshad M, et al. Global survey of genetic variation in CCR5, RANTES, and MIP-1alpha: impact on the epidemiology of the HIV-1 pandemic. Proc Natl Acad Sci U S A. 2001;98(9):5199-5204.

43. Li K, Dai D, Yao L, et al. Association between the macrophage inflammatory protein-1 alpha gene polymorphism and Alzheimer's disease in the Chinese population. Neurosci Lett. 2008;433(2):125-128.

44. Man SM, Ma YR, Shang DS, et al. Peripheral T cells overexpress MIP1alpha to enhance its transendothelial migration in Alzheimer's disease. Neurobiol Aging. 2007;28(4):485-496.

45. Winkler C, An P, O'Brien SJ. Patterns of ethnic diversity among the genes that influence AIDS. Hum Mol Genet. 2004;13 Spec No 1: R9-R19.

46. Antinori A, Arendt G, Becker JT, et al. Updated research nosology for HIV-associated neurocognitive disorders. Neurology. 2007;69(18): 1789-1799.
Neurobehavioral HIV Medicine

\section{Publish your work in this journal}

Neurobehavioral HIV Medicine is an international, peer-reviewed, open access journal focusing on advances in research in HIV/AIDS, with specific reference to the neurological, psychiatric and behavioral consequences of the disease, concomitant infections and specific antiretrovira therapy. The manuscript management system is completely online and

\section{Dovepress}

includes a very quick and fair peer-review system, which is all easy to use. Visit http://www.dovepress.com/testimonials.php to read real quotes from published authors. 